\title{
PROBLEMATIC INTERNET USE AMONG ADOLESCENTS AND THE VIEW OF CONTEXT: A PLS-STRUCTURAL EQUATION MODEL
}

\author{
Lucrezia Ferrante, Claudia Venuleo, \& Simone Rollo \\ Department of History, Society and Human Studies, University of Salento (Italy)
}

\begin{abstract}
The idea of Internet use as a way to face psychosocial malaise is growing in the scientific literature about Problematic Internet Use (PIU). The present study, assuming the Semiotic Cultural Psycho-social Theory (SCPT) (Salvatore, 2018) as theoretical framework, postulates and emphasizes that the context in which the subject is embedded provide the symbolic resources, which ground the way adolescents perceive, experience, and therefore deal with the material and social world, including the likelihood of using the Internet as a way to facing life problems and difficulties. SCTP adopts the term "Symbolic Universes" (SU) to denote affect-laden assumptions concerning the world which may (or not) promote adaptive responses.

Specifically, the present study aimed to test a mediation model in which each Symbolic Universes (i.e. independent variable) is associated with the psychosocial malaise in terms of social anxiety, loneliness, and negative emotions (i.e. mediator variable), which in turn has effects on PIU (i.e. dependent variable).

Measures of PIU (GPIUS), symbolic universes (VOC), negative affect (PANAS), social anxiety (IAS), loneliness (ILs) among a total of 764 Southern Italy youths aged from 13 to 19 (mean age =15.05 \pm 1.152). A Multiple Correspondence Analysis (MCA) was firstly run to detect SU; a Partial Least Squares Structural Equation Modelling (PLS-SEM) was then performed on R for testing the hypothesized mediation model.

The results demonstrated that Symbolic Universes characterized by anomie and unreliability of the social context are associated with adolescents' PIU though the mediation of social anxiety, loneliness, and negative emotions.

Overall, findings suggest that within an anomic and unreliable scenario, PIU might acquire the meaning of a way to face life in an environment that seems meaningless, uncertain, and detrimental. On the plane of intervention, this points to the need for programs that address social and cultural influences in youths' Internet use.
\end{abstract}

Keywords: Problematic internet use, adolescents, symbolic universes, social environment, psychosocial malaise.

\section{Introduction}

The negative effects of Internet use among adolescents have gained increasingly attention in the last decades. Although the lack of a widely accepted definition of Problematic Internet Use (PIU) (Musetti et al., 2016; Spada, 2014), it is well-recognized that it may occur when Internet use works as a way of escaping from problems and gaining relief (Young, 1998), coping with difficult life experiences (Griffiths, 2005), or regulating moods (Caplan, 2010). Therefore, for a better understanding of PIU among adolescents, it seems important to consider their life problems which may explain its onset and maintenance.

The present study, assuming the Semiotic Cultural Psycho-social Theory (SCPT) (Salvatore, 2018) as theoretical framework, postulates and emphasizes that the context in which the subject is embedded provide the symbolic resources, which ground the way adolescents perceive, experience, and therefore deal with the material and social world, including the likelihood of using the Internet as a way to face life problems and difficulties. SCTP adopts the term "Symbolic Universes" to denote basic intuitive, embodied, affect-laden assumptions concerning the world - what it is and how it works - which may (or not) promote adaptive responses. Each Symbolic Universe can be interpreted as the result the foregrounding of a certain set of generalizing meanings encompassing the experience as a whole (e.g. "life is a question of luck"), while others are left in the background (e.g. "life depends on efforts and 
pain"); SU is not set in the head of the individual, rather it is the by-product of a continuous intersubjective negotiation within people's life spheres (e.g. family, school, workplace) mediated by the symbolic resources provided by the cultural context.

Previous studies have showed the relationship between the ways by which people interpret their social experience and their attitude toward problematic/hazardous behaviors consistently with the tenet that the meanings any SU is composed of are ways of experiencing the social environment, which foster and constrain actions and reactions. For instance, studies among problem gamblers, alcoholics and problematic internet users (Venuleo et al., 2016) have found that they differ from controls due to a negative view of the social environment, perceived as anomic, unreliable, and destined not to change at all.

According to SPCT, the capacity of Symbolic Universes to promote adaptive responses is a function of two aspects (Venuleo et al., 2020): 1) the consistency between the beliefs, feelings and actions underpinned by the Symbolic Universes, and the role demands made on the subjects by the social environment, that is whether the Symbolic Universe encourage behaviors and attitudes that are related to interpersonal and social tasks, rules and goals; 2) the variable degree of salience of the generalised meanings composing them, with a high salience corresponds to a rigid, polarised, way of thinking, producing homogenising affect-laden interpretations of the reality (typically organized by the bad/good, pleasure/displeasure opposition) and reducing the capacity to regulate thoughts, desires and beliefs, and to tackle social constraints and requirements effectively.

\subsection{Aim and hypothesis}

Based on the SCPT and the view of PIU as a way to face psychosocial malaise, the present study aims to test a theoretical model model in which each Symbolic Universes is associated with the psychosocial malaise in terms of social anxiety, loneliness, and negative emotions, which in turn has effects on PIU. We expect that a negative view of the social environment - underpinned by a low consistency with social tasks, rules and goal as well as homogenising affect-laden interpretations - may fuel negative affect (e.g. sadness, stress, loneliness, and other intolerable feelings) which in turn increase the need to indulge in Internet use: indeed, it is reasonable that within a view of their own social network as poor and untrustworthy, Internet use can seem as the only way to cope with problems in life.

\section{Method}

\subsection{Participants and instruments}

The study was performed in public high schools situated in the Southern Italy with a total of 764 students attending yr. $9(52 \%)$ and $11(48 \%)$ and aged from 13 to 19 (mean age = 15.05 $\pm 1.152)$. Participants were asked to complete an online survey containing the following scales:

a. The Generalized Problematic Internet Use Scale - 2 (GPIUS-2; Caplan, 2010), 15 items associated to eight points Likert scale (from "Definitely disagree" to "Definitely agree") $(\alpha$ Cronbach $=.91)$;

b. View of Context (VOC; Ciavolino et al., 2017), 45 items associated with a four-points Likert scale (from "Not at all" to "A lot" or from "Very unreliable" to "Very reliable") ( $\alpha$ Cronbach $=.79$ );

c) The Interaction Anxiousness Scale (IAS; Leary, 1983), 15 items associated to five points Likert scale (from "Not at all characteristic of me" to "Extremely characteristic of me") $(\alpha$ Cronbach $=.66)$;

d) The Negative Affect subscale of the Positive and Negative Affect Schedule (PANAS; Watson et al., 1988), 10 items associated to 5-point Likert scale (from "Not at all" to "Very much") $(\alpha$ Cronbach $=.80)$;

c) General Loneliness Sub-scale from Italian Loneliness Sub-scale (ILS; Zammuner, 2008), 7 items associated to four points Likert scale (from "I often feel this way" to "I never feel this way") $(\alpha$ Cronbach $=.85)$.

\subsection{Data analysis}

Firstly, to detect Symbolic Universes, a Multiple Correspondence Analysis (MCA) was computed on items of View of Context questionnaire. Factorial dimensions were extracted from this analysis, with each factor describing the juxtaposition of two opposite models of response modes (i.e. "generalized meanings") which organize (dis)similarities in the way of interpreting the social environment. The MCA provides a measure of the degree of association of any respondent with every factorial dimension, expressed in terms of respondent's position (coordinate) on the factorial dimension. Accordingly, the SU the student belongs to has been detected in terms of two factorial coordinates - one 
for each factor/dimension of sense extracted and interpreted based on the largest proportion of the data matrix inertia explained. This analysis was run on the software SPAD.

Secondly, the Partial Least Squares Structural Equation Modelling (PLS-SEM) was performed on $\mathrm{R}$ software for testing two hypothesized mediation models, one for each Symbolic Universe as independent variable, and both with the psychosocial malaise as mediator variable, and PIU as dependent variable.

\section{Results}

\subsection{Symbolic universes}

The first factorial dimension of the VOC (Symbolic Universe 1) accounts for $48.62 \%$, and the second (Symbolic Universe 2) for $16.41 \%$, thus the two factors account for $65.03 \%$ of the total inertia (Benzécri, 1979). Symbolic Universe 1 opposes two patterns of answers that have been interpreted as the markers of two models of relationship with one's own social environment: Agency (-) versus Anomie (+). On the Agency polarity, answers adopting intermediate choices on the Likert scales are aggregated (e.g. "somewhat agree", "somewhat disagree"), referring to the possibility of determining life with one's own actions, to the importance of understanding the world, and in disagreement with fatalistic attitudes (life depends on luck, it is not possible to predict the future), in a context of sufficiently trustworthy institutions. On the Anomie polarity, answers adopting extreme choices are aggregated (e.g. "strongly agree", "not at all"), referring to the importance of not being scrupulous and be allied with the strongest, the impossibility to count on anyone and for people to change, the absence of faith in the development of the country, and an unreliable vision of services and institutions. Symbolic Universe 2 opposes two patterns of answers that have been interpreted as the markers of two way of evaluating the social environment: Absolute reliability (-) versus Moderate unreliability (+). On the Absolute reliability polarity answers adopting extreme choices on the Likert scales are aggregated (e.g. "very", "much better"), referring to school, police, public administration as trustworthy, to faith in a better future and in the possibility that people change, and to the importance of following the rules, sharing, and acquiring knowledge. On the Moderate unreliability polarity answers adopting intermediate choices on the Likert scales are aggregated (e.g. "not very", "somewhat"), referring to an almost absent trust in services and institutions, as well as a weak faith in the future of the country and in the possibility to succeed in life following the rules and acquiring knowledge.

\subsection{The mediation PLS-SEM model}

In Model 1 with Symbolic Universe 1 "Model of relationship with the social environment" as independent variable, the Anomie polarity was significantly positively related to psychosocial malaise, and psychosocial malaise was significantly positively related to PIU. In Model 2 with Symbolic Universe 2 "Evaluation of the social environment" as independent variable, the Unreliability polarity was significantly positively related to psychosocial malaise, and psychosocial malaise was significantly positively related to PIU. In both cases, the results support the quality of the measurement models with Cronbach alpha and Dillon-Goldstein's rho indices of 0.70 and higher, indicating homogeneity of the indicators, and the eigen values $>1$, revealing the unidimensionality of the model.

Figure 1. PLS-SEM with Symbolic Universe 1 - Anomie as independent variable.

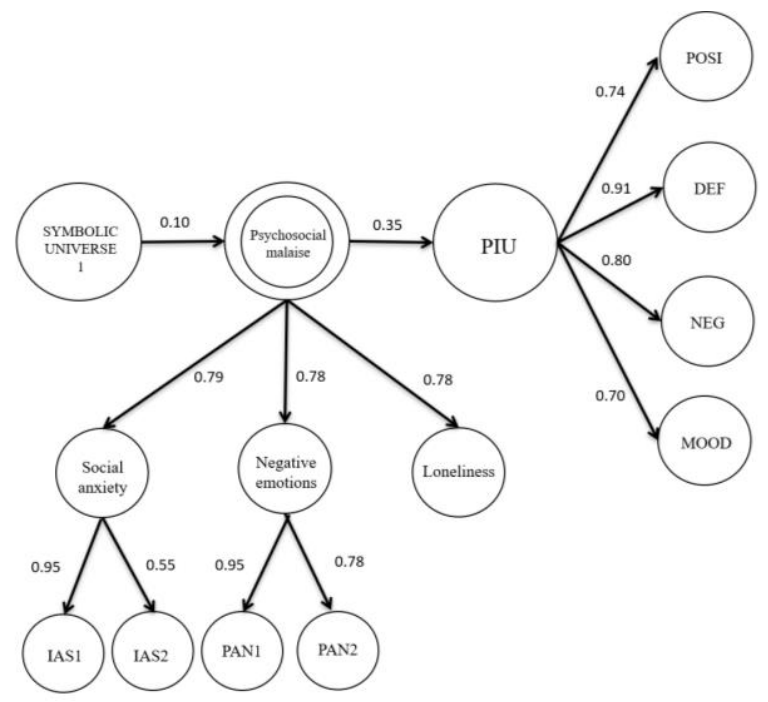


Figure 2. PLS-SEM with Symbolic Universe 2 - Moderate unreliability of the system as independent variable.

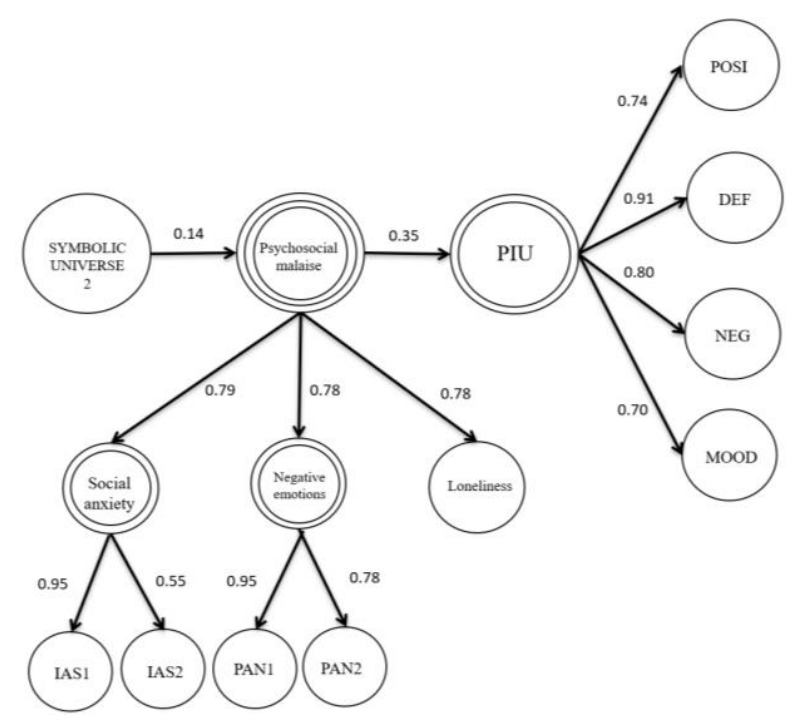

\section{Discussion}

Our results support that Symbolic Universes are associated with adolescents' PIU though the mediation of psychosocial malaise.

Particularly, adolescents tending to adopt the Anomie polarity of the Symbolic Universes "Models of relationship with the social environment" are more likely to manifest malaise which, in turn, is associated to PIU: they perceived their socio-cultural environment as a place where people cannot absolutely count on anyone, and breaking rules, not having scruples and being allied with the strongest is the only way to get by; there is no faith at all in the future or even in people, institutions and services. They showed rigid, polarized, and extremely negative connotations and evaluations of their environment: the reality is interpreted in a homogenising way not considering the variability of objects or events of the experience. According to our view, such reactive adolescents' tendency to homogenize their experience and express extremely negative connotations and evaluations can be interpreted as the marker of an intense affective activation - not unlike a person that is very upset will tend to see everything around them as triggers for anger -, which, from a clinical standpoint, hampers the ability to use reality for the regulation of one's thoughts, desires and beliefs, therefore to face social constraints and requirements effectively (Salvatore \& Freda, 2011).

Findings also showed that high psychosocial malaise in our sample is associated to the position on the Moderate unreliability polarity of the Symbolic Universe "Evaluation of the social environment", that is the polarity referring to lack of trust in institutions (e.g. schools, the Church, police) and services (e.g. health departments, public offices), as well as a weak adherence to beliefs such as the importance of sharing, acquiring knowledge and following rules. This point seems consistent with our standpoint that the role of the Symbolic Universes in promoting distress depends also on degree to which individuals' beliefs, feelings, and actions, underpinning it, encourage or not behaviors compatible with interpersonal and social tasks, rules and goal. In this sense, adolescents in our study who do not seem to easily find in their environment anyone (i.e. institutions and people) or anything (i.e. tenets, models, beliefs) to rely on and to follow, reported higher psychosocial malaise, which increases the risk of PIU.

Overall, our findings provided support to the idea that a negative view of the social environment, perceived as anomic and unreliable, is associated to psychosocial malaise (social anxiety, loneliness, negative emotions) which in turn enhances the need to overindulge in Internet use as a way to face feelings and situations that are felt to be unbearable. Such interpretation is consistent with the view of PIU as a maladaptive mechanism for compensating individual's problems; it worth to highlight that the present study further contributes to understand the development of PIU highlighting that the malaise which adolescents try to face with Internet can be understood as a reflex of of the way they interpret their social experience, namely Symbolic Universes. In this sense, Internet is used in a rigid, persistent and maladaptive way (i.e. problematic) to compensate unpleasant states within a context perceived as lacking of support, opportunities, something or someone to rely on, for facing life distress

With regard clinical implications, considering the role of sense-making in PIU development and maintenance, suggest that any intervention that restrict its action to the specific behaviours (e.g. to inform adolescents and children about how to use Internet in order to remove negative outcomes related to its 
utilization) is likely to have limited efficacy, given that the way people feel, think, and act is function of their global worldview that concerns the world of experience as a whole. By this perspective, risk prevention is more radically, a matter of cultural meanings to interpret their own social experience. On the plane of intervention, this points to the need for comprehensive programming of prevention and health promotion that addresses multiple influences in the various contexts of adolescents' lives (Thorlindsson, 2011): family-focused interventions may be more effective than youth-only focused prevention services, and culture-focused interventions addressing parents and adolescents' cultural assumptions should improve interventions success even more (Kumpfer et al., 2002).

\section{References}

Benzecri, J.P. (1979). Sur le calcul des taux d'inertie dans l'analyse d'un questionnaire. Cahiérs Anal Données, 4, 377-378.

Caplan, S. E. (2010). Theory and measurement of generalized problematic Internet use: A two-step approach. Computers in Human Behavior, 26(5), 1089-1097.

Ciavolino, E., Redd, R., Evrinomy, A., Falcone, M., Fini, V., Kadianaki, I., ... \& Rochira, A. (2017). Views of Context. An instrument for the analysis of the cultural milieu. A first validation study. Electronic Journal of Applied Statistical Analysis, 10(2), 599-628.

Griffiths, M. (2005). A "components" model of addiction within a biopsychosocial framework. Journal of Substance Use, 10(4), 191-197.

Kumpfer, K. L. (2002). Factors and processes contributing to resilience. In Resilience and development (pp. 179-224). Boston, MA: Springer.

Leary, M. R. (1983). Social anxiousness: The construct and its measurement. Journal of personality assessment, 47(1), 66-75.

Musetti, A., Cattivelli, R., Giacobbi, M., Zuglian, P., Ceccarini, M., Capelli, F., ... \& Castelnuovo, G. (2016). Challenges in internet addiction disorder: is a diagnosis feasible or not?. Frontiers in psychology, 7, 842 .

Salvatore, S. (2018). Culture as dynamics of sense-making. A semiotic and embodied framework for sociocultural psychology. In J. Valsiner, \& A. Rosa (Eds), Cambridge Handbook of Culture \& Psychology. Cambridge, UK: Cambridge Handbooks in Psychology.

Salvatore, S., \& Freda, M. F. (2011). Affect, unconscious and sensemaking. A psychodynamic, semiotic and dialogic model. New ideas in psychology, 29(2), 119-135.

Spada, M. M. (2014). An overview of problematic Internet use. Addictive behaviors, 39(1), 3-6.

Thorlindsson, T. (2011). Bring in the social context: Towards an integrated approach to health promotion and prevention. Scandinavian journal of public health, 39 (6 suppl), 19-25.

Venuleo, C., Rollo, S., Marinaci, T., \& Calogiuri, S. (2016). Towards a cultural understanding of addictive behaviours. The image of the social environment among problem gamblers, drinkers, internet users and smokers. Addiction Research \& Theory, 24(4), 274-287.

Venuleo, C., Salvatore, G., Andrisano-Ruggieri, R., Marinaci, T., Cozzolino, M., \& Salvatore, S. (2020). Steps towards a unified theory of psychopathology: The Phase Space of Meaning model. Clinical Neuropsychiatry, 17(4), 236-252.

Watson, D., Clark, L. A., \& Tellegen, A. (1988). Development and validation of brief measures of positive and negative affect: the PANAS scales. Journal of personality and social psychology, 54(6), 1063

Young, K. S. (1998). Internet addiction: The emergence of a new clinical disorder. Cyberpsychology \& behavior, 1(3), 237-244.

Zammuner, V. L. (2008). Italians' social and emotional loneliness: The results of five studies. International Journal of Social Sciences, 3(2), 108-120. 